\title{
MMC controlled-release membranes attenuate epidural scar formation in rat models after laminectomy
}

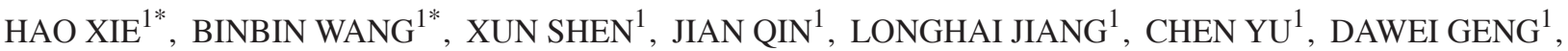 \\ TANGBO YUAN $^{1}$, TAO WU ${ }^{2}$, XIAOJIAN CAO ${ }^{3}$ and JUN LIU ${ }^{1,2}$ \\ ${ }^{1}$ Department of Orthopaedics, Sir Run Run Hospital, Nanjing Medical University; ${ }^{2}$ Department of Orthopaedics, \\ The Second Affiliated Hospital of Nanjing Medical University; ${ }^{3}$ Department of Orthopaedics, \\ The First Affiliated Hospital of Nanjing Medical University, Nanjing, Jiangsu 210000, P.R. China
}

Received February 13, 2017; Accepted March 27, 2017

DOI: $10.3892 / \mathrm{mmr} .2017 .6531$

\begin{abstract}
Epidural scar formation after laminectomy impede surgical outcomes of decompression. Mitomycin C (MMC) has been demonstrated to have significant inhibitory effects on epidural scar. This study was undertaken to develop an effective MMC controlled-release membrane and to investigate its effects on epidural scar in rat models of laminectomy. A total of 72 rats that underwent laminectomy were divided into three groups. Among them, 24 were treated with mitomycin C-polylactic acid (MMC-PLA) controlled-release membrane, 24 with mitomycin C-polyethylene glycol (MMC-PEG) controlled-release membrane, and no treatment was performed for the remaining 24 rats (control group). In the following 4 weeks, magnetic resonance image (MRI), macroscopic observation, histology and hydroxyproline (Hyp) concentration analysis were performed to explore the effects of these three therapies on epidural scar. MRI revealed a significant reduction of epidural fibrosis in MMC-PLA and MMC-PEG treatment groups, compared with the control group. Histological results also showed that collagen deposition was significantly reduced after being treated with MMC-PLA or MMC-PEG membranes. Likewise, Hyp concentrations of the epidural scar tissue in MMC-PLA and MMC-PEG groups were markedly lower than those in the control group. However, regarding the effects on reducing epidural scar, no significant difference was found between the MMC-PLA and MMC-PEG groups. In conclusion, MMC-PLA and MMC-PEG
\end{abstract}

Correspondence to: Professor Jun Liu, Department of Orthopaedics, The Second Affiliated Hospital of Nanjing Medical University, 121 Jiang Jiayuan Road, Drum Tower, Nanjing, Jiangsu 210000, P.R. China

E-mail: liujun112900@163.com

${ }^{*}$ Contributed equally

Key words: mitomycin C, polylactic acid, polyethylene glycol, epidural scar, laminectomy membranes are safe and effective in reducing fibrosis. Thus, MMC-controlled-release membranes promises to be a potential therapeutic in preventing epidural scar formation after laminectomy.

\section{Introduction}

Postoperative fibrosis is a natural consequence of surgical wound healing and epidural fibrosis is an expected healing consequence after laminectomy. The source of fibrotic tissue after spinal surgery is originally thought to arise from the disrupted intervertebral disc and the disrupted epaxial muscles in the surgical wound $(1,2)$. This extradural fibrotic tissue may extend to the vertebral canal and adhere to the dura mater and nerve roots, causing recurrent symptoms including pain (3-5), possibly leading to the failure of spinal surgery (6). Although there remains controversy about the role of peridural fibrosis in failed back surgery syndrome $(7,8)$, it has been widely accepted that it is a problematic clinical entity with no efficacious treatment options (9-11). Furthermore, epidural adhesions make re-exposure of the same operative area technically difficult and dangerous because of the greatly increased risk in nerve root injury and dural tears $(12,13)$.

The control of scar formation has been one of the main concerns in spine surgery. Numerous types of biological or non-biological materials and methods for preventing epidural fibrosis have been developed, including autologous fat grafts, absorbable gelatin films and sponges, protein-based polymer, high-molecular weight hyaluronan, collagen, gelatin foam $(14,15)$, which have been implanted to serve as a barrier $(16,17)$ to physically or chemically inhibit scar ingrowths.

Polylactic acid (PLA) and polyethylene glycol (PEG) are water-soluble polymers without any electric charge and affinity for any specific organ, and are non-immunogenic and non-toxic $(18,19)$. They exhibit good biological and physicochemical properties. Due to their non-toxic, biodegradable and hydrophilic characteristics, PLA and PEG have been widely used in tissue engineering $(18,19)$. Although PLA and PEG significantly reduce post-laminectomy proliferative scar without any impact on the integrity of incisional wound 
healing, the process of re-absorption will result in a fibrotic mass and form a gap between the sheet and the dura $(20,21)$. Moreover, PLA and PEG could not prevent nerve root adhesion.

Because of its capacity to inhibit DNA-dependent RNA synthesis (22), mitomycin C (MMC), a well-known chemotherapeutic agent, has been proposed as a potential adjuvant for the control of scar tissue in surgical wounds and used successfully for a long time to prevent scar formation and fibrosis $(23,24)$.

In the present study, we used two control-release delivery films, in which a small dose of MMC $(0.01 \%)$ was absorbed in the PLA and PEG films. In our preliminary studies, we established a stable controlled-release delivery system (25). There are many studies on the suppressing effect of MMC on the post-laminectomy scar invasion, but there few studies on its repairing effect on the insignificant cerebrospinal fluid leaks. The purpose of our research was to investigate and compare the efficacy of MMC-PLA and MMC-PEG films as a biophysical and chemical barrier to suppress post-laminectomy scar invasion. Another purpose of our investigation was to assess whether the barrier film contained MMC would influence the dural spontaneous healing causing cerebrospinal fluid leakage.

We hypothesized that with the decomposition of PLA and PEG films, MMC would be continuously released to inhibit scar ingrowths without affecting the healing of the dura. If true, the barrier film containing a low concentration of MMC is safe to be used for spinal surgery without affecting the healing of the cerebrospinal fluid leaks. Particularly, it is well-adapted for the re-exposure of the same operative area.

\section{Materials and methods}

All the procedures were performed with the permission of the Ethics Committee of Nanjing Medical University, and all the rats were obtained from the Animal Experimental Research Center of Jiangsu Province. A total of 72 male Sprague-Dawley rats weighing $400+\mathrm{g}$ were used for the study, which were divided into three groups. Group 1 ( $n=24$, the control group): only the standard surgical procedure was performed (L5 total laminectomy, bilateral disk injury and central dura injury); group 2 ( $n=24)$ : the mitomycin C-polylactic acid (MMC-PLA) controlled-release film group, in which the barrier film served as a roof tenting over the space between the ronguered edges of the laminectomy area, and group $3(n=24)$ : the mitomycin C-polyethylene glycol (MMC-PEG) controlled-release film group, in which the application of barrier film was the same as group 2 .

Surgical procedure. All the procedures were performed by the same surgeon applying a standard surgical procedure on each rat. A posterior midline incision was made from L4 to L7 vertebrae to expose the bony posterior elements and carried sharply down to the lumbosacral fascia. With blunt dissection, the paraspinal musculature was subperiosteally dissected and the lumbar vertebral segments were exposed. Keeping the spinal cord and cauda equina intact, bilateral laminectomies were performed at L5-L6, and the dura mater and nerve roots were exposed. The neural elements and dura were retracted gently and sufficiently medially to expose the posterolateral aspect of the L5-L6 disc. Then a 26-gauge needle was inserted into the bilateral exposed L5-L6 disc, creating a disc injury. Next, with the help of a 10 times microscope, an incision was made into the dura and subarachnoid space (durotomy) by an 18-gauge needle, creating a dura mater injury. The durotomy was considered complete when cerebrospinal fluid was observed leaking outside the dura.

In the control group, the lumbosacral fascia and other layers were sutured directly. In the experimental groups (the MMC-PLA and MMC-PEG groups), after creating a disc injury and a dura mater injury, the barrier film was cut into a suitable size ( $2 \times 2 \mathrm{~cm}, 25-30 \mathrm{mg}$ PLA or PEG film containing $0.01 \% \mathrm{MMC}$ ) and then applied as a roof tenting over the space between the ronguered edges of the laminectomy area. Then the lumbosacral fascia and other layers were sutured.

No other medical treatment that could increase or reduce the potential effects of the agents was used. The rats were closely followed up during the first $24 \mathrm{~h}$ for the neurologic status. The standard diet and the same conditions were provided for each rat. All the rats were followed up for 4 weeks. We observed the postoperative recovery of all rats and evaluated whether there were neurologic deficits or cerebrospinal leak. The rats were sacrificed on the 28 th day after operation by a lethal dose of pentobarbital.

Magnetic resonance image (MRI) examination. MRI examination was carried out at the 4 th week after surgery. Six rats were selected from each group randomly. After general anesthesia, the whole spine columns from L4 to L7 including the surrounding muscle tissues were dissected and removed to perform the MRI examination. The MRIs were acquired by Bruker 7.0T micro-MR imaging system (Siemens Corp., Berlin, Germany) and Multi-Slice Multi-Echo T2-weighted imaging (MSME T2WI) sequence. Two sets of MR images were performed: they were spin echo T1-weighted images in sagittal and transverse planes (TR_2710 ms, TE_22 ms) and the gradiently recalled echo $\mathrm{T} 2$-weighted images in the same locations with spin echo images for sagittal and transverse images (TR_4391 ms, TE_33 ms). For each imaging slice encompassing the operative level, the amount of epidural fibrosis was graded on the scale of 0-4: grade 0, no scar/trace of a scar; grade $1,>0$ to $\leq 25 \%$ of the quadrant filled with scar; grade $2,>25$ to $\leq 50 \%$ of the quadrant filled with scar; grade 3, $>50$ to $\leq 75 \%$ of the quadrant filled with scar; and grade 4 , $75-100 \%$ of the quadrant being filled with scar (26). Briefly, the spinal canal in each level was further subdivided into four quadrants by drawing perpendicular lines from the central aspect of the dura sac. Three radiologists analyzed the MR images of epidural fibrosis independently with ImageJ software (version X; Media Cybernetics, Silver Springs, MD, USA).

Macroscopic observation of the epidural scar adhesion. Macroscopic evaluation was performed at the 4 th week after operation. A total of 18 rats were randomly selected from the remaining rats (6 from each group). Three pathologists observed the epidural scar tissue and determined the degree of epidural adhesion according to the Rydell standard independently. We observed the rats simultaneously to assure whether there was a leakage of cerebrospinal fluid from the durotomy site. All the pathologists were blinded to the 
Table I. Pathological analysis scoring system of the peridural fibrosis and adhesion.

\begin{tabular}{ll}
\hline Grade & \multicolumn{1}{c}{ Pathological features } \\
\hline Grade 0 & $\begin{array}{l}\text { The dura is free of scar tissue } \\
\text { Presence of only thin fibrous bands between } \\
\text { dura and scar tissue }\end{array}$ \\
Grade 2 & $\begin{array}{l}\text { Continuous adherence between dura and } \\
\text { scar tissue involving }<2 / 3 \text { of laminectomy defect } \\
\text { Scar tissue adherence } \geq 2 / 3 \text { of laminectomy } \\
\text { defect and/or extend to nerve roots }\end{array}$ \\
\hline
\end{tabular}

Table II. Fibroblast cell density scoring system.

\begin{tabular}{lc}
\hline Grade & Cell density \\
\hline Grade 1 & $<100$ cells in each area at $x 400$ \\
Grade 2 & $100-150$ cells in each area at $x 400$ \\
Grade 3 & $>150$ cells in each area at $x 400$ \\
\hline
\end{tabular}

groups. The degree of epidural adhesion was graded on the scale of 0-3: Grade 0, epidural scar tissue was not adherent to the dura mater; Grade 1, epidural scar tissue was adherent to the dura mater, but easily dissected; Grade 2, epidural scar tissue was adherent to the dura mater and difficult to dissect without disrupting the dura mater; Grade 3, epidural scar tissue was firmly adherent to the dura mater and could not be dissected.

Histological analysis. Histological analysis was performed at the 4 th week after surgery. A total of 18 rats were randomly selected from remaining rats ( 6 from each group). Subsequently, the spinal columns, including surrounding muscle tissue, were resected. All specimens were harvested to assess the whole anatomy of fibrosis formation on the space between the dura mater and the surrounding soft tissues. The specimens were cut and ground into $\sim 100$-micron axial sections. All the sections were stained with hematoxylin and eosin and Masson's trichrome, and then photographed by a microscope (BX-42; Olympus, Tokyo, Japan). Two histopathologists with similar qualifications, who were blinded and independent to the treatment, evaluated and measured the peridural fibrosis and the fibroblast cell density according to the classification (Table I and II) described by Sen et al (26).

Biochemical analysis. Another six rats were randomly selected from each group at the 4 th week after operation. The scar tissue, about $0.2 \times 0.2 \times 0.5 \mathrm{~cm}$, was harvested from the space between the dura mater and surrounding soft tissues from each rat. The dissected tissues were rinsed, homogenated, centrifuged, and hydrolyzed for the measurement of hydroxyproline (Hyp). The Hyp developer was then added into the digested solution. The absorbance was read by a spectrophotometer (Hitachi, Tokyo, Japan) at $550 \mathrm{~nm}$, and the contents of Hyp per milligram tissue were calculated.
Table III. Grade of epidural scar adhesion according to the Rydell standard.

\begin{tabular}{lllll}
\hline & \multicolumn{4}{c}{ Grade } \\
\cline { 2 - 5 } Group & 0 & 1 & 2 & 3 \\
\hline Control & 0 & 0 & 0 & 6 \\
${ }^{a}$ MMC-PLA & 4 & 2 & 0 & 0 \\
bMMC-PEG & 5 & 1 & 0 & 0
\end{tabular}

${ }^{\mathrm{a}} \mathrm{P}<0.05$, the control group compared with MMC-PLA group. ${ }^{\mathrm{b}} \mathrm{P}<0.05$, the control group compared with MMC-PEG group. There was no significant difference between the experimental groups. MMC-PLA, mitomycin C-polylactic acid; MMC-PEG, mitomycin C-polyethylene glycol.

Statistical analysis. The statistical analysis was performed by the SPSS 19.0 (SPSS, Inc., Chicago, IL, USA) statistical package. Data were presented as means \pm standard deviations. The differences between groups were evaluated with the non-parametrical test (Mann-Whitney U test). P-values $<0.05$ were considered statistically significant.

\section{Results}

In all rats, the postoperative recovery was uneventful except for two with skin infection (one rat in the control group, the other in the polylactic acid (PLA) film group. The infections were superficial and controlled with povidone-iodine, without using antibiotics. There was no case with neurologic deficits or cerebrospinal leak in any of the rats. No rats suffered accidental death.

MRI evaluation. Transverse MRI obtained at the laminectomy level of the control group rats exhibited noticeable fibrotic scarring compression in the spine cord (Fig. 1A). However, the experimental groups showed a discrete hypo-signal space between the dura mater and the surrounding fibrosis tissue near the skin (Figs. 1B and C).

The MRI score demonstrated that it was higher in the control group when compared with that in the experimental groups $(\mathrm{P}<0.05)$. However, there was no significant difference between experimental groups ( $\mathrm{P}>0.05$, Fig. 1D).

Macroscopic observation of the epidural scar adhesion. Macroscopic observation by two independent evaluators showed the soft or weak fibrosis adhesion around the laminectomy areas in the experimental groups, which could be easily dissected by manual traction and clean dura mater was exposed without any evident adhesion or membrane. Leakage of cerebrospinal fluid from the durotomy site was not observed in any of the rats in the experimental groups. But in the laminectomy sites of rats in the control group, there was a severe epidural scar tissue disrupting dura mater around the laminectomy areas. Severe, thick, and tenacious epidural scar adhesions were found between the dura mater and surrounding tissue. Complete re-exposure of the dura mater was impossible for the possibility to cause serious bleeding and the risk of nerve 
A

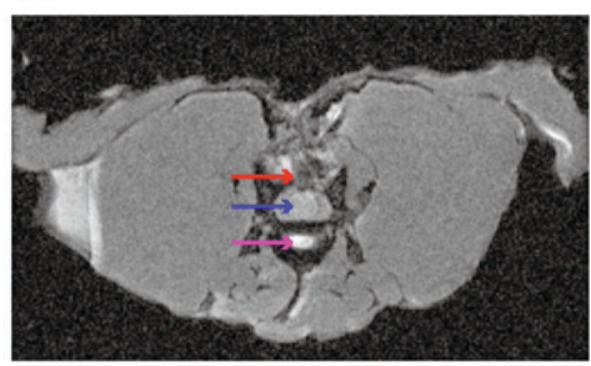

C

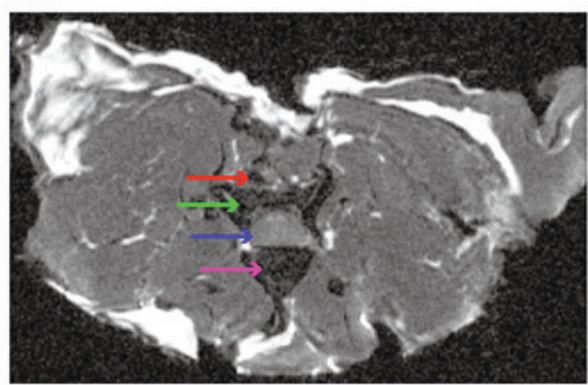

B

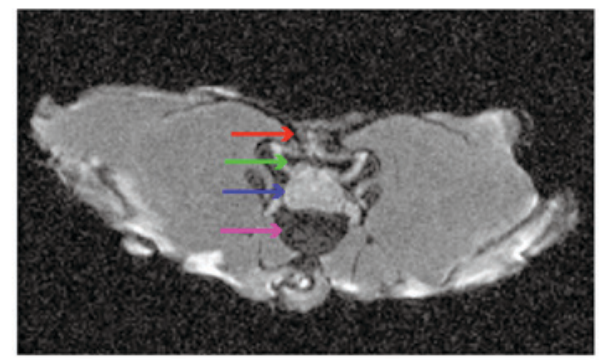

$\mathrm{D}$

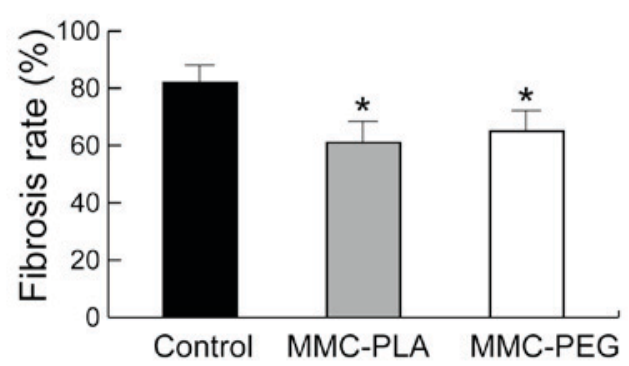

Figure 1. Transverse MR image of the laminectomy site in different groups at 4 weeks post-surgery. (A) Control-group. (B) MMC-PLA group. (C) MMC-PEG group. (D) Comparison of the epidural fibrosis score calculated from MR images. The epidural fibrosis score was significantly decreased in the experimental rats compared with that in the control group rats at 4 weeks post-surgery. Red arrow, noticeable fibrotic scarring; blue arrow, compression in the spine cord; purple arrow, intervertebral disc or vertebral region; green arrow, a discrete hypo-signal space between the dura mater and the surrounding fibrosis tissue near the skin; "P<0.05 compared with the control group. MMC-PLA, mitomycin C-polylactic acid; MMC-PEG, mitomycin C-polyethylene glycol.
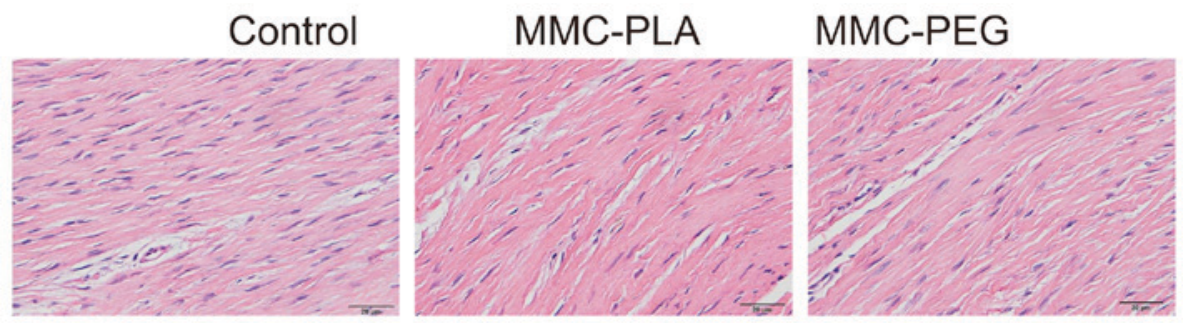

Figure 2. Photomicrographs of the epidural adhesion issues in the laminectomy sites in the control and experimental groups. There was dense scar tissue adhered to dura mater in the control group. Loose scar tissues without adherence to dura mater were present in the experimental group. MMC-PLA, mitomycin C-polylactic acid; MMC-PEG, mitomycin C-polyethylene glycol.
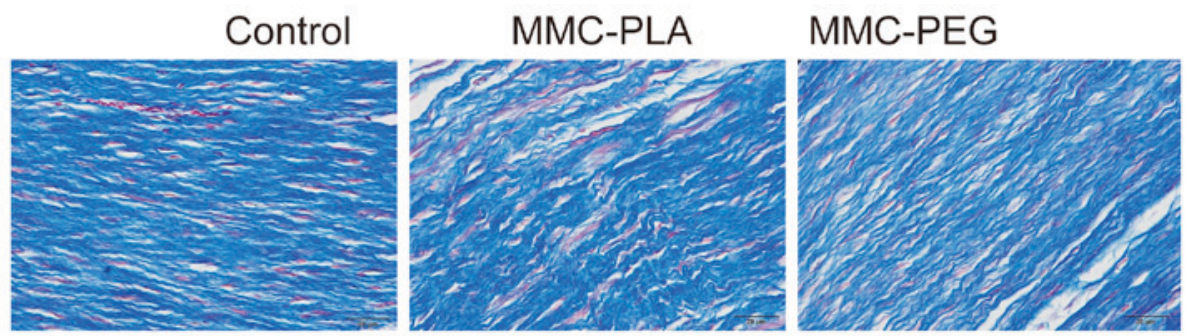

Figure 3. Photomicrographs of the epidural adhesion issues in the laminectomy sites in the control and experimental groups. Representative histological images of collagen tissue proliferation in epidural scar tissue of rats not treated in the control group, and MMC-PLA and MMC-PEG in the experimental groups. The collagen tissues show blue in the section stained with Masson's trichrome. The density of collagen tissue in the section treated with MMC-PLA or MMC-PEG was significantly less than that in the control group. MMC-PLA, mitomycin C-polylactic acid; MMC-PEG, mitomycin C-polyethylene glycol.

root injury or dura tears. The grades of epidural adhesion in rats according to the Rydell standard are shown in Table III.

Histological analysis of epidural adhesion. In the control group, marked epidural scar tissue with widely spread adhesions to dura mater and dorsal aspect fascia or muscle was noted primarily in the laminectomy sites. Dense scar tissue mixed with extensive collagen tissue hyperplasia was observed. There were also abundant fibroblasts and fibrocytes in the laminectomy sites. 


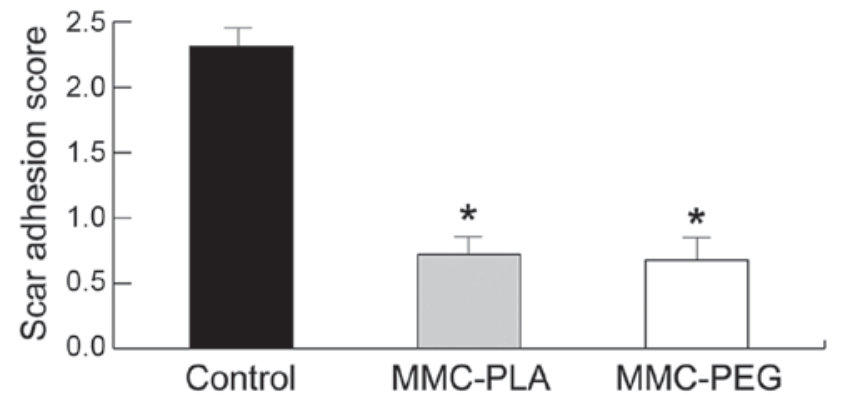

Figure 4. The results of epidural scar adhesion scoring in epidural scar tissue based on histological evaluation in each group. ${ }^{*} \mathrm{P}<0.05$, compared with the control group. MMC-PLA, mitomycin C-polylactic acid; MMC-PEG, mitomycin C-polyethylene glycol.

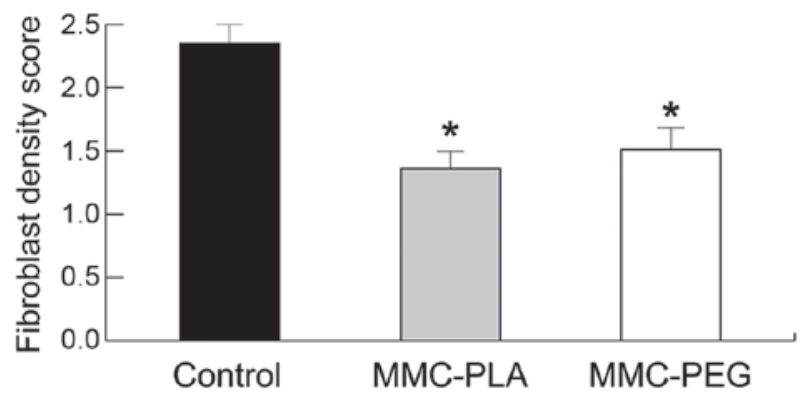

Figure 5. The results of fibroblast cells density scoring in epidural scar tissue based on histological analysis in each group. ${ }^{*} \mathrm{P}<0.05$, compared with the control group.

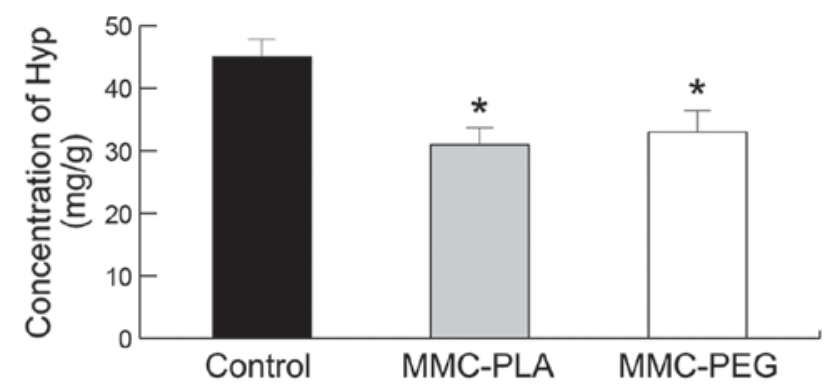

Figure 6. The varied Hyp concentration of epidural scar tissue from different groups. Significant difference was observed between the experimental and control groups. ${ }^{*} \mathrm{P}<0.05$, compared with the control group. Hyp, hydroxyproline; MMC-PLA, mitomycin C-polylactic acid; MMC-PEG mitomycin C-polyethylene glycol.

In the experimental groups, loosely arranged epidural scar tissue with no or little dura adhesion to the dura mater and dorsal aspect fascia or muscle was discovered. We could also observe that the dura mater was slightly thickened and it was separated from the epidural scar tissue through an empty interspace. The collagen tissue hyperplasia was obviously decreased (Figs. 2 and 3).

Inflammation was not observed in any of the laminectomy sites in any of the rats. Peridural fibrosis and adhesions were evaluated and summarized in Fig. 4. Fibroblast cell density was evaluated and summarized in Fig. 5.

Biochemical analysis. The Hyp concentration in the epidural scar tissue in the experiment groups (MMC-PLA and
MMC-PEG) was significantly reduced compared with that in the control group $(\mathrm{P}<0.05$, Fig. 6). The precise concentrations of each group were as follows: the control group, 45.2132.53 mg/g; MMC-PLA, 31.4157.21 mg/g; MMC-PEG, $33.6736 .91 \mathrm{mg} / \mathrm{g}$.

\section{Discussion}

The present study demonstrated that the MMC-PLA and the MMC-PEG controlled-release delivery barrier films could be implanted as a physical and chemistry barrier to effectively reduce epidural fibrosis formation and scar adhesion without affecting the cerebrospinal fluid leaks healing. The results provided sufficient evidence that the MMC-PLA and the MMC-PEG controlled-release delivery barrier films were a potential biomaterial to reduce epidural fibrosis for future clinical application.

In MRI evaluation, the transverse MRI of the control group exhibited noticeable fibrotic scarring compression in the spine cord, and the experimental groups had a lower MRI score $(\mathrm{P}<0.05)$. In macroscopic observation of the epidural scar adhesion, the grade of the control group was 3 compared with the experimental group grades from 0 to $1(\mathrm{P}<0.05)$. In histological analysis of epidural adhesion, there was a dense scar tissue adhered to dura mater in the control group, and loose scar tissues without adherence to dura mater were present in the experimental group. The density of collagen tissue in the section treated with MMC-PLA or MMC-PEG was significantly less than that in the control group. In biochemical analysis, the Hyp concentration in the epidural scar tissue in the experiment groups (MMC-PLA and MMC-PEG) was significantly reduced compared with that in the control group $(\mathrm{P}<0.05)$. Also, there was no case of neurologic deficits or cerebrospinal leakage in any rat. No rat suffered accidental death.

The study demonstrated that with the decomposition of PLA and PEG films, MMC would be continuously released and inhibited the scar ingrowths without affecting the healing of the dura.

Various reagents and materials have been used to prevent epidural fibrosis after laminectomy. Several different strategies have been extensively explored. Microsurgical technique (27), drug use $(28,29)$ and low-dose radiation $(30-32)$ have been demonstrated to be effective in rat and human models. At the same time, there are also studies on the biophysical barriers which could be applied to avoid the contact between the epidural scar and dura $(33,34)$.

Bioresorbable PLA and PEG barrier films are promising and currently used in surgical scenarios (35-37). They are used for temporary wound support to reinforce soft tissue, and minimize soft tissue attachments in the viscera by preventing acute platelet deposition on damaged arteries and reducing adhesion of platelets and leukocytes to the surface (35-37).

Both films could impede the migration of fibroblasts from the raw surface of the erector spine musculature. They also act as a surgical dissection plane, whereby the layer of organized fibrous tissue enveloping the material forms a controlled, distinguishable dissection plane allowing adjacent tissues to be easily separated $(35,38)$. 
However, the 'three-dimensional theory', put forward by Songer et al (39), suggested that the PLA and PEG films could not function as a three-dimensional barrier.

Compared with the PLA or PEG films, MMC could leak into every space in the operation area and has been widely applied as a potential adjuvant for the control of scar tissue in surgical wounds $(22,23,40)$. However, the toxic characteristics of MMC are most likely responsible for the greater tissue damage causing increased scar tissue formation and even the failure of the wound healing (41).

In most cases, insignificant cerebrospinal fluid leaks during lumbar surgery are spontaneously repaired with the help of fibroblasts or fibrocytes. Nevertheless, because the barrier film prevents fibroblast migration and MMC prevents fibroblast proliferation, these small leaks may do not heal spontaneously and cerebrospinal fluid leaks develop (42).

In our study, compromised dura healing or cerebrospinal fluid leaks were not identified with any of the test products in the rats using MRI examination, macroscopic observation, histological and biochemical analyses. The unique feature of our study is that we used a controlled-release delivery system in which a small dose of MMC was absorbed into the PLA and PEG films to prepare the MMC-PLA and MMC-PEG films attempting to prevent the epidural scar adhesions from developing cerebrospinal fluid leaks. In our study, the two mitomycin C control-release films of PEG and PLA barrier could function as a three-dimensional barrier. At the same time, as only a small dose of MMC was applied, the MMC controlled-release film should not interrupt the integrity of the spontaneous repairing of the dura and give rise to some other troublesome side effects. We, therefore, believe that the use of MMC controlled-release film represents a better way of creating a barrier to the expansion of the troublesome epidural fibrosis.

In conclusion, the present study suggests that the two mitomycin-C controlled-release barrier films of PEG and PLA is an effective and safe material to decrease epidural scar adhesion after spinal laminectomy in the rat model. Our findings indicate that applying the mitomycin- $\mathrm{C}$ controlled-release barrier films in clinical practice may potentially minimize post-operative complications.

\section{Acknowledgements}

This study was supported by the National Natural Science Foundation of China (grant nos. 81301523 and 81271987) and and Jiangsu High Level Medical Personnel '111111 Project Reasearch' (grant no. LGY2016018).

\section{References}

1. Key JA and Ford LT: Experimental intervertebral-disc lesions. J Bone Joint Surg Am 30A: 621-630, 1948.

2. Yang L, Tang J, Chen H, Ge D, Sui T, Que J, Cao X and Ge Y: Taurine reduced epidural fibrosis in rat models after laminectomy via downregulating EGR1. Cell Physiol Biochem 38: 2261-2271, 2016.

3. Bartynski WS and Petropoulou KA: The MR imaging features and clinical correlates in low back pain-related syndromes. Magn Reson Imaging Clin N Am 15: 137-154, 2007.

4. Sizer PS Jr, Phelps V, Dedrick G and Matthijs O: Differential diagnosis and management of spinal nerve root-related pain. Pain Pract 2: 98-121, 2002.
5. Lladó A, Guimerá J, Garcia F and Navarro A: Expanded polytetrafluoroethylene membrane for the prevention of peridural fibrosis after spinal surgery: An experimental study. Eur Spine J 8: 138-143, 1999.

6. Burton CV: Causes of failure of surgery on the lumbar spine: Ten-year follow-up. Mt Sinai J Med 58: 183-187, 1991.

7. Ganzer D, Giese K, Völker L, Pietzner U, Follak N and Merk H: Two-year results after lumbar microdiscectomy with and without prophylaxis of a peridural fibrosis using Adcon-L. Arch Orthop Trauma Surg 123: 17-21, 2003.

8. Vogelsang JP, Finkenstaedt M, Vogelsang M and Markakis E: Recurrent pain after lumbar discectomy: The diagnostic value of peridural scar on MRI. Eur Spine J 8: 475-479, 1999.

9. Ozgen S, Naderi S, Ozek MM and Pamir MN: Findings and outcome of revision lumbar disc surgery. J Spinal Disord 12: 287-292, 1999.

10. Samy Abdou M and Hardy RW Jr: Epidural fibrosis and the failed back surgery syndrome: History and physical findings. Neurol Res 21 (Suppl 1): S5-S8, 1999.

11. BenDebba M, Augustus van Alphen H and Long DM: Association between peridural scar and activity-related pain after lumbar discectomy. Neurol Res 21 (Suppl 1): S37-S42, 1999.

12. Chandler K and Cappello R: Laminectomy membrane formation in dogs: Is the answer still elusive? Vet J 172: 1-2, 2006.

13. Ming J, Wei T, Lin C, Min T, Sheng W and Chih Y: Spinal somatosensory evoked potential to evaluate neurophysiologic changes associated with postlaminotomy fibrosis: An experimental study. Spine 32: 2111-2118, 2007.

14. da Costa RC, Pippi NL, Graça DL, Fialho SA, Alves A, Groff AC and Rezler U: The effects of free fat graft or cellulose membrane implants on laminectomy membrane formation in dogs. Vet J 171: 491-499, 2006.

15. Kato T, Haro H, Komori $\mathrm{H}$ and Shinomiya K: Evaluation of hyaluronic acid sheet for the prevention of postlaminectomy adhesions. Spine J 5: 479-488, 2005.

16. Temel SG, Ozturk C, Temiz A, Ersozlu S and Aydinli U: A new material for prevention of epidural fibrosis after laminectomy: Oxidized regenerated cellulose (interceed), an absorbable barrier. J Spinal Disord Tech 19: 270-275, 2006.

17. Akeson WH, Massie JB, Huang B, Giurea A, Sah R, Garfin SR and Kim CW: Topical high-molecular-weight hyaluronan and a roofing barrier sheet equally inhibit postlaminectomy fibrosis. Spine J 5: 180-190, 2005.

18. Pasut G, Guiotto A and Veronese FM: Protein, peptide and non-peptide drug PEGylation for therapeutical application (Review). Expert Opin Ther Patents 14: 859-894, 2004.

19. Schiavon O, Pasut G, Moro S, Orsolini P, Guiotto A and Veronese FM: PEG-Ara-C conjugates for controlled release. Eur J Med Chem 39: 123-133, 2004.

20. Tao H and Fan H: Implantation of amniotic membrane to reduce postlaminectomy epidural adhesions. Eur Spine J 18: 1202-1212, 2009.

21. Cao B, Yin J, Yan S, Cui L, Chen X and Xie Y: Porous scaffolds based on cross-linking of poly(L-glutamic acid). Macromol Biosci 11: 427-434, 2011.

22. Dogulu F, Kurt G, Emmez H, Erdem O, Memis L, Baykaner K and Ceviker N: Topical mitomycin C-induced inhibition of postlaminectomy peridural fibrosis in rabbits. J Neurosurg 99 (Suppl 1): 76-79, 2003.

23. Sun Y, Wang LX, Wang L, Sun SX, Cao XJ, Wang P and Feng L: A comparison of the effectiveness of mitomycin C and 5-fluorouracil in the prevention of peridural adhesion after laminectomy. J Neurosurg Spine 7: 423-428, 2007.

24. Lee JY, Stenzel W, Löhr M, Stützer H, Ernestus RI and Klug N: The role of mitomycin $\mathrm{C}$ in reducing recurrence of epidural fibrosis after repeated operation in a laminectomy model in rats. J Neurosurg Spine 4: 329-333, 2006.

25. Liu J, Ni B, Zhu L, Yang J, Cao X and Zhou W: Mitomycin Cpolyethylene glycol controlled-release film inhibits collagen secretion and induces apoptosis of fibroblasts in the early wound of a postlaminectomy rat model. Spine J 10: 441-447, 2010.

26. Sen O, Kizilkilic O, Aydin MV, Yalcin O, Erdogan B, Cekinmez M, Caner $\mathrm{H}$ and Altinors N: The role of closed-suction drainage in preventing epidural fibrosis and its correlation with a new grading system of epidural fibrosis on the basis of MRI. Eur Spine J 14: 409-414, 2005.

27. Canis M, Botchorishvili R, Tamburro S, Safi A, Wattiez A, Mage G, Pouly JL and Bruhat MA: Adhesion prevention in the surgical treatment of pelvic endometriosis. Gynaecol Endosc 10: 99-106, 2001. 
28. Zhang Z, Tarone G and Turner DC: Expression of integrin $\alpha 1 \beta 1$ is regulated by nerve growth factor and dexamethasone in PC12 cells. Functional consequences for adhesion and neurite outgrowth. J Biol Chem 268: 5557-5565, 1993.

29. Dumont RJ, Verma S, Okonkwo DO, Hurlbert RJ, Boulos PT, Ellegala DB and Dumont AS: Acute spinal cord injury, part II: Contemporary pharmacotherapy. Clin Neuropharmacol 24 265-279, 2001.

30. Gerszten PC, Moossy JJ, Flickinger JC, Gerszten K, Kalend A and Martínez AJ: Inhibition of peridural fibrosis after laminectomy using low-dose external beam radiation in a dog model. Neurosurgery 46: 1478-1485, 2000.

31. Gerszten PC, Moossy JJ, Bahri S, Kalend A and Martínez AJ: Inhibition of peridural fibrosis after laminectomy using low-dose external beam radiation in a rat model. Neurosurgery 44 : 597-602, discussion 602-603, 1999.

32. Gibbs IC, Patil C, Gerszten PC, Adler JR Jr and Burton SA: Delayed radiation-induced myelopathy after spinal radiosurgery. Neurosurgery 64 (suppl_2): A67-A72, 2009.

33. Pospiech J, Pajonk F and Stolke D: Epidural scar tissue formation after spinal surgery: An experimental study. Eur Spine J 4: 213-219, 1995.

34. Silva SS, Motta A, Rodrigues MT, Pinheiro AF, Gomes ME, Mano JF, Reis RL and Migliaresi C: Novel genipin-cross-linked chitosan/silk fibroin sponges for cartilage engineering strategies. Biomacromolecules 9: 2764-2774, 2008.

35. Klopp LS, Welch WC, Tai JW, Toth JM, Cornwall GB and Turner AS: Use of polylactide resorbable film as a barrier to postoperative peridural adhesion in an ovine dorsal laminectomy model. Neurosurg Focus 16: E2, 2004.
36. Bakaltcheva I, Ganong JP, Holtz BL, Peat RA and Reid T: Effects of high-molecular-weight cryoprotectants on platelets and the coagulation system. Cryobiology 40: 283-293, 2000.

37. Fujii H, Fujii S, Togashi H, Yoshioka M, Nakai K, Satoh H, Sakuma I, Kenmotsu O and Kitabatake A: Attenuation of hypothermia-induced platelet activation and platelet adhesion to artificial surfaces in vitro by modification of hemoglobin to carry S-nitric oxide and polyethylene glycol. Thromb Res 100: 519-528, 2000

38. Welch WC, Cornwall GB, Toth JM, Turner AS, Thomas KA, Gerszten PC and Nemoto EM: Use of polylactide resorbable film as an adhesion barrier. Orthopedics 25 (10 Suppl): s1121-s1130, 2002.

39. Songer MN, Rauschning W, Carson EW and Pandit SM: Analysis of peridural scar formation and its prevention after lumbar laminotomy and discectomy in dogs. Spine 20: 571-580, discussion 579-580, 1995.

40. Lee JY, Stenzel W, Ebel H, Wedekind C, Ernestus RI and Klug N: Mitomycin C in preventing spinal epidural fibrosis in a laminectomy model in rats. J Neurosurg 100 (1 Suppl Spine): 52-55, 2004.

41. Fielding JW, Crocker J, Stockley RA and Brookes VS: Interstitial fibrosis in a patient treated with 5-fluorouracil and mitomycin $\mathrm{C}$. BMJ 2: 551-552, 1979

42. Kuhn J, Hofmann B, Knitelius HO, Coenen HH and Bewermeyer H: Bilateral subdural haematomata and lumbar pseudomeningocele due to a chronic leakage of liquor cerebrospinalis after a lumbar discectomy with the application of ADCON-L gel. J Neurol Neurosurg Psychiatry 76: 1031-1033, 2005. 